\title{
Layered Capacity-Based Relay-and-Antenna Joint Selection for MIMO-AF-Multiple-Relay Systems in Correlated Channels
}

\author{
Chia-Chang Hu, Jheng-Siang Li, and Bo-Liang Wu \\ Department of Communications Engineering, National Chung Cheng University, Min-Hsiung, \\ Chiayi 62102, Taiwan \\ Correspondence should be addressed to Chia-Chang Hu, ieecch@ccu.edu.tw
}

Received 1 June 2012; Revised 2 August 2012; Accepted 2 August 2012

Academic Editor: Jung-Chieh Chen

Copyright (๑) 2012 Chia-Chang Hu et al. This is an open access article distributed under the Creative Commons Attribution License, which permits unrestricted use, distribution, and reproduction in any medium, provided the original work is properly cited.

\begin{abstract}
A computationally efficient two-stage greedy capacity maximization (GCM) relay-and-antenna joint selection is proposed for a dual-hop nonregenerative amplify-and-forward (AF) multiple-input multiple-output (MIMO) multiple-relay system with multiple antennas equipped at each node in correlated fading channels. This modified GCM (MGCM) antenna selection strategy selects a subset of antenna pairs from available relays based on the concept of channel capacity maximization subject to an optimal power allocation constraint across the activated antenna pairs. In order to reduce system hardware complexity, antenna selection schemes are performed at the destination node as well. Finally, simulations are conducted to compare the channel capacity of the proposed two-layered antenna selection technique with other existing antenna selection algorithms for half-duplex AF-MIMO multiple-relay systems.
\end{abstract}

\section{Introduction}

Multiple-input multiple-output (MIMO) systems using multielement antennas (MEA) at the transmitter and the receiver improve substantially capacity and reliability of wireless links $[1,2]$. As a simplified version of spatial multiplexing (SM) systems, the vertical Bell Laboratories layered space-time (V-BLAST) architecture [3] exploits the channel capacity and diversity gain of MEA systems over the richscattering wireless channel. A new communication paradigm that incorporates MIMO technologies into cooperative relay networks $[4,5]$ has attracted significant interest recently. In the cooperative communications and networking, the source node communicates with the destination node by making use of wireless relays. With the aid of node cooperation, wireless relay networks are able to obtain spatial diversity in a distributed manner. In general, there are two categories of cooperative relaying strategies, such as nonregenerative amplifyand-forward- (AF-) relaying protocols $[5,6]$ and regenerative decode-and-forward- (DF-) relaying protocols [5, 7].
An AF-mode protocol shows a simple relaying approach and possesses a low implementation complexity. However, the limitation on the implementation of MIMO systems is the cost and complexity of multiple radio frequency (RF) chains associated with multiple antennas. The increase in system hardware and signal processing complexity has inhibited the widespread adoption of MIMO systems. Fortunately, the utilization of antenna subset selection approaches for MIMO systems has successfully become a low-cost alternative due to the reduced complexity without significant performance degradation compared to the full-complexity (FC) MIMO system [8]. These approaches alleviate system hardware complexity by using fewer RF chains than the actual number of antenna elements.

So far most of antenna selection techniques applied to non-regenerative wireless relay networks are focusing on single-antenna relay models [9] or single-stream MIMOrelay networks [10]. An opportunistic relaying approach, namely, distributed orthogonal relay selection (DORS), for dual-hop noncoherent AF-MIMO relay networks are 
proposed in [11] based on the harmonic mean of dualhop subchannel gains. In [12], a cross-entropy- (CE-) based method is introduced to perform the optimal relay subset selection for two-way AF-MIMO relaying systems in order to reduce computational complexity while still maximizing the achievable sum rate. However, only single-antenna relays are dealt with for one-or two-way dual-hop AF-wireless MIMOrelay networks. A fast antenna subset selection algorithm (FASSA) [13] is proposed for two-hop AF- and DF-MIMO relay systems to maximize system capacity. The selection criterion of the FASSA algorithm for AF relay is designed to maximize a lower bound of the capacity, but not the exact capacity. The authors in [14] present a two-stage antenna subset selection scheme to maximize the channel capacity of a conventional three-node AF-MIMO relay system. Moreover, a low-complexity near-optimal antenna selection algorithm is considered based on a constrained cross-entropy optimization (CCEO) method to maximize the achievable rate for MIMO-AF single-relay networks in [15]. However, only single relay is dealt with in a nonregenerative multiantenna relay network. In [16], a greedy mean squared error (MSE) minimization antenna selection is developed under an equal-power allocation across the multistream data for AF-MIMO relay systems. The fact that only single-antenna pair can be activated on each relay node in the greedy MSE-based minimization (GMM) antenna selection should be noted. Additionally, the computational complexity of the GMM antenna selection remains high and is thus of limited practical use. A greedy capacity maximization (GCM) antenna selection technique proposed in [17] is applied to an identical scenario employed in [16]. Four joint relay and antenna selection strategies are investigated in terms of the outage probability in [18] with the use of either full or partial CSI for dual-hop MIMO-AF multiple-relay networks. A comprehensive performance analysis framework is provided based on practical transmission impairments in [19] for dual-hop MIMO-AF relay networks with hopby-hop beamforming (i.e., both source and relay perform beamforming). In [20], the outage probability and the average symbol-error-rate (SER) performance of three transmit antenna selection (TAS) strategies are analyzed for dual-hop MIMO ideal channel-assisted AF-relay networks. Although these antenna selection schemes are effective, most of these works have not taken spatial correlation between antennas into account. Various measurements have shown that the realistic MIMO-channel capacity is significantly lower than that in the ideal case owing to the spatial correlation of the MIMO channel. The correlation between the signal transmitted/received at different antenna elements is due to the lack of spacing between antennas as well as the existence of small angular spread [21]. Therefore, to mitigate the effect of spatial correlation, a correlated channel model has to be employed to replace the ideal identically independent distribution (iid.) one.

In the paper, a reduced-complexity two-stage relay-andantenna joint selection is proposed for a dual-hop nonregenerative AF-MIMO multiple-relay system with multiple antennas equipped at each node over correlated fading channels. This relay-and-antenna selection strategy selects a subset of antenna pairs from multiple relays based on channel capacity maximization under an optimal power allocation constraint across the activated antenna pairs. To reduce further system hardware and signal processing complexity, antenna selection schemes are also performed at the destination node. In addition, all wireless channel links are characterized as spatially correlated random variables based on the Kronecker correlation model [22]. As a consequence, the use of the proposed two-layer relay-and-antenna joint selection is capable of trading in a relatively small fraction of loss in system performance, as compared with existing antenna selection schemes for half-duplex AF-MIMO relay networks, for a considerable reduction in system hardware and signal processing complexity.

The remainder of the paper is organized as follows. Section 2 describes the system and channel model. Section 3 presents a two-stage modified GCM (MGCM) antenna selection technique for a dual-hop non-regenerative MIMOAF multiple-relay system in correlated channels. Both the optimal and equal-power allocation constraints across the activated $K$ antenna pairs in the half-duplex AF-MIMO multiple-relay system are provided in Section 4. To further alleviate hardware cost and implementation complexity of a dual-hop AF-wireless MIMO multiple-relay system, destination antenna selection schemes are utilized and analyzed in Section 5. Two kinds of existing relay-and-antenna joint selection criteria are briefly reviewed in Section 6. Numerical results and conclusions are presented in Sections 7 and 8, respectively.

Notation 1. Symbols for matrices (vectors) are denoted by boldface upper- (lower-) case letters. The superscripts $(\cdot)^{\top},(\cdot)^{\mathrm{H}}$, and $(\cdot)^{-1}$ are transposition, Hermitian transposition, and matrix inversion operations, respectively. $\|\cdot\|_{\mathrm{F}}$ indicates the matrix/vector Frobenius norm. $E\{\cdot\}$ represents the expected-value operator. $\mathbf{I}_{M}$ is an $M \times M$ identity matrix. $\operatorname{tr}(\cdot)$ and $[\cdot]_{i, j}$ denote, respectively, the trace and the $(i, j)$ th entry of a matrix. $(\cdot)^{1 / 2}$ is the square root of a positive semidefinite matrix. Finally, $(x)_{+}$stands for $\max (x, 0)$.

\section{System and Channel Model}

A dual-hop non-regenerative AF-wireless MIMO multiplerelay system is considered in correlated fading channels, as illustrated in Figure 1. The information-bearing signals are sent from an $N_{\mathrm{S}}$-antenna source terminal to an $N_{\mathrm{D}}$-antenna destination terminal with the aid of $N$ half-duplex relay terminals each with $N_{\mathrm{R}}$ antennas. Note that no direct link is considered due to the long distance or the fading obstacle between the source and the destination terminals. In order to provide sufficient degrees of freedom for signal detection, the condition of $N_{S} \leq \min \left\{N_{\mathrm{R}}, N_{\mathrm{D}}\right\}$ is preserved in what follows. In addition, elements of all channel links such as the source-to-relay (backward) channels $\mathrm{H}_{\mathrm{SR}_{i}} \in C^{N_{\mathrm{R}} \times N_{\mathrm{S}}}$ and the relay-to-destination (forward) channels $\mathrm{G}_{\mathrm{R}_{i} \mathrm{D}} \in C^{N_{\mathrm{D}} \times N_{\mathrm{R}}}$ for all $i \in[1, N]$ are modeled as spatially correlated random 


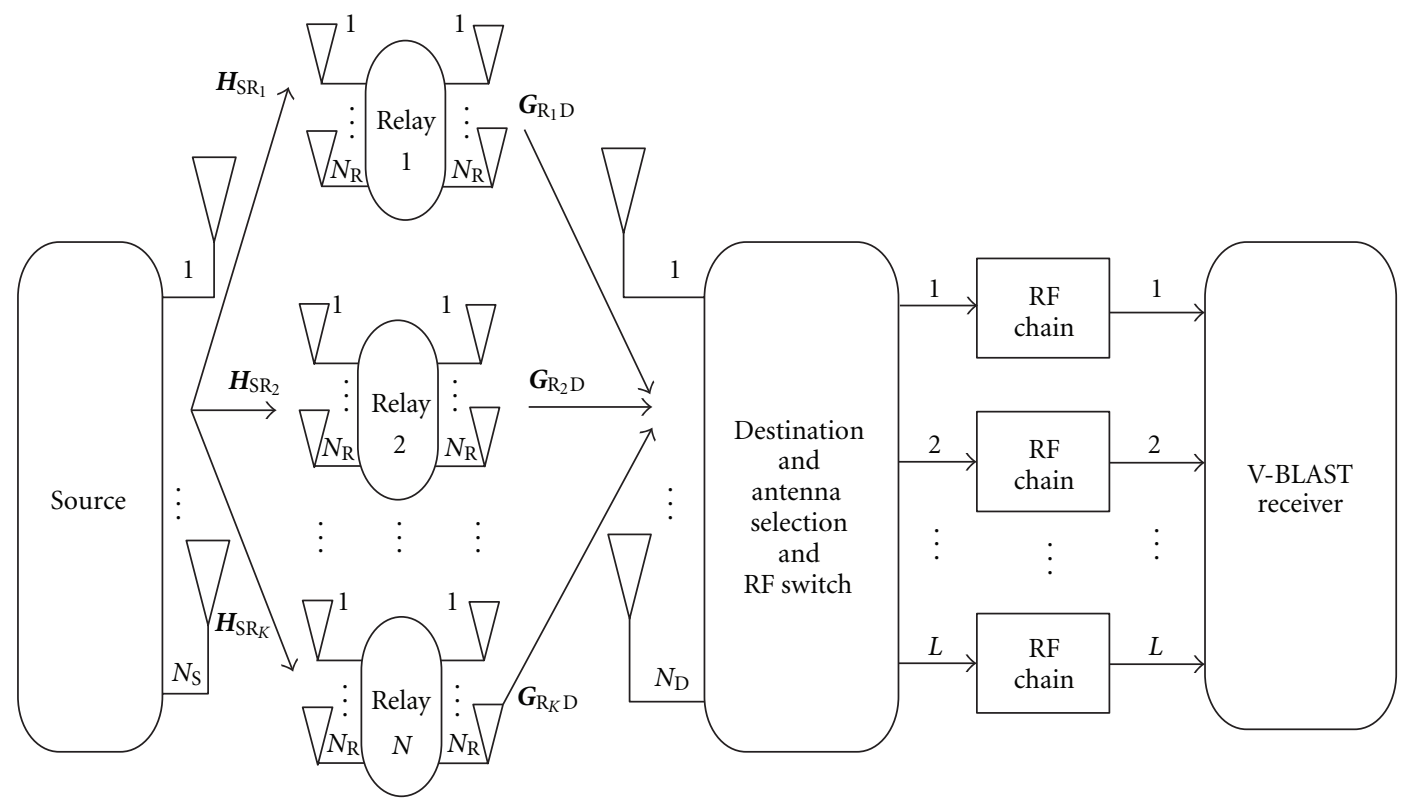

Figure 1: Block diagram of an AF-approach MIMO multiple-relay system in which the source node, each of $N$ half-duplex AF-relay nodes, and the destination node are equipped with $N_{\mathrm{S}}, N_{\mathrm{R}}$, and $N_{\mathrm{D}}$ antennas, respectively.

variables based on the Kronecker correlation model [22], which are given as follows:

$$
\begin{array}{r}
\mathbf{H}_{\mathrm{SR}_{i}}=\Sigma_{\mathrm{SR}_{i}}^{1 / 2} \mathbf{H}_{\mathrm{SR}_{i}}^{w} \Psi_{\mathrm{SR}_{i}}^{1 / 2}=\left[\mathbf{h}_{i, 1}^{\top}, \mathbf{h}_{i, 2}^{\top}, \ldots, \mathbf{h}_{i, N_{\mathrm{R}}}^{\top}\right]^{\top}, \\
i=1,2, \ldots, N_{\mathrm{S}}, \\
\mathbf{G}_{\mathrm{R}_{i} \mathrm{D}}=\Sigma_{\mathrm{R}_{i} \mathrm{D}}^{1 / 2} \mathbf{G}_{\mathrm{R}_{i} \mathrm{D}}^{w} \Psi_{\mathrm{R}_{i} \mathrm{D}}^{1 / 2}=\left[\mathbf{g}_{i, 1}, \mathbf{g}_{i, 2}, \ldots, \mathbf{g}_{i, N_{\mathrm{R}}}\right], \\
i=1,2, \ldots, N_{\mathrm{D}},
\end{array}
$$

where $\mathbf{H}_{\mathrm{SR}_{i}}^{w} \in C^{N_{\mathrm{R}} \times N_{\mathrm{S}}}$ and $\mathrm{G}_{\mathrm{R}_{\mathrm{i}} \mathrm{D}}^{w} \in C^{N_{\mathrm{D}} \times N_{\mathrm{R}}}$ are, respectively, the backward and the forward spatially white complex Gaussian random matrices, in which elements are i.i.d. zeromean circularly symmetric complex Gaussian (ZMCSCG) random variables with unit covariance. $\boldsymbol{\Sigma}_{\mathrm{SR}_{i}} \in C^{N_{\mathrm{R}} \times N_{\mathrm{R}}}$ and $\boldsymbol{\Sigma}_{\mathrm{R}_{\mathrm{D}} \mathrm{D}} \in C^{N_{\mathrm{D}} \times N_{\mathrm{D}}}$ are the receive-side spatial correlation matrices and $\Psi_{\mathrm{SR}_{i}} \in C^{N_{\mathrm{S}} \times N_{\mathrm{S}}}$ and $\Psi_{\mathrm{R}_{\mathrm{i}} \mathrm{D}} \in C^{N_{\mathrm{R}} \times N_{\mathrm{R}}}$ are the transmit-side correlation matrices for the backward channel $\mathbf{H}_{\mathrm{SR}_{i}}$ and the forward channel $\mathbf{G}_{\mathrm{R}_{i} \mathrm{D}}$, respectively. $\mathbf{h}_{i, j}$ and $\mathbf{g}_{i, j}$, $j=1,2, \ldots, N_{\mathrm{R}}$, represent, respectively, the $1 \times N_{\mathrm{S}}$ backward channel vector from the source node to the $j$ th antenna of the $i$ th relay and the $N_{\mathrm{D}} \times 1$ forward channel vector from the $j$ th antenna of the $i$ th relay to the destination node.

In what follows, it should be noted that the correlation between different MIMO-channel elements in the Kronecker channel model is modeled under the assumption that the correlation among receive antennas is independent of the correlation between transmit antennas. The approximation of the spatial cross-correlation function $r(d)$, that determines the correlation between two adjacent antenna elements separated in space by a distance $d$ [23], is given as follows:

$$
r(d) \approx \exp \left[-23 A^{2}\left(\frac{d}{\lambda}\right)^{2}\right]
$$

where $\lambda$ is the wavelength and $A$ is the angular spread parameter. The angular spread parameter of $A$ is defined by [24]

$$
A=\frac{\sqrt{\theta^{2}+2 \cos \theta-2}}{2 \theta},
$$

where $\theta \in[0,2 \pi]$ denotes the width of the sector of arriving multipath power.

During the first time slot, a subset of $K$ antennas are selected from $N$ relay nodes each with $N_{\mathrm{R}}$ antennas, that is, a total of $N N_{\mathrm{R}}$ antenna selection choices. In the sequel, let the subscript of the pair $(R(k), b(k)), k=1,2, \ldots, K$, represent the $k$ th selected antenna index $b(k)$ and the corresponding relay index $R(k)$ in time-slot I. The $K$-activated antenna pairs generate a $K \times N_{\mathrm{S}}$ compound backward channel matrix $\mathbf{H}$ given by

$$
\mathbf{H}=\left[\mathbf{h}_{(R(1), b(1))}^{\top}, \mathbf{h}_{(R(2), b(2))}^{\top}, \ldots, \mathbf{h}_{(R(K), b(K))}^{\top}\right]^{\top} .
$$

The received signal vector at the $K$ antenna pairs can be expressed as

$$
\mathbf{y}=\left[y_{1}, y_{2}, \ldots, y_{K}\right]^{\top}=\mathbf{H} \mathbf{x}+\mathbf{n}_{\mathrm{R}},
$$

where $y_{i}=\mathbf{h}_{(R(i), b(i)) \mathbf{x}}+n_{i}, i=1,2, \ldots, K$, is the $i$ th received signal component in $\mathbf{y}$ and $n_{i}$ is the corresponding noise random variable in $\mathbf{n}_{\mathrm{R}}$ during the first-time slot. In (5), $\mathbf{x} \in C^{N_{S} \times 1}$ stands for the information-bearing signal vector whose elements are chosen independently from the same constellation and satisfy $\mathbf{R}_{\mathbf{x}}=E\left\{\mathbf{x x}^{\mathrm{H}}\right\}=\sigma_{\mathrm{x}}^{2} \mathbf{I}_{N_{\mathrm{S}}}$ and $\mathbf{n}_{\mathrm{R}} \in C^{K \times 1}$ denotes an i.i.d. ZMCSCG noise vector with the covariance matrix $\mathbf{R}_{\mathbf{n}_{\mathrm{R}}}=E\left\{\mathbf{n}_{\mathrm{R}} \mathbf{n}_{\mathrm{R}}^{\mathrm{H}}\right\}=\sigma_{n_{\mathrm{R}}}^{2} \mathbf{I}_{K}$. Additionally, the power constraint at the source is written as $E\left\{\operatorname{tr}\left(\mathbf{x x}^{\mathrm{H}}\right)\right\} \leq P_{\mathrm{S}}$. Under the case of the equal power allocation, $\sigma_{\mathrm{x}}^{2}=P_{\mathrm{S}} / N_{\mathrm{S}}$ is 
attained. In the second-time slot, the received signal vector $\mathbf{y}$ is amplified by a $K \times K$ relay weighting matrix $\mathbf{W}$ and subsequently the relay forwards the precoded signal vector to the destination. The amplified signal vector denoted by $\mathbf{p}$ is given by

$$
\mathrm{p}=\mathrm{Wy}
$$

Note that the total transmit power of $K$ activated antenna pairs selected from $L_{\mathrm{R}}$ relays is bounded by $P_{\mathrm{R}}$ as follows:

$$
E\left\{\operatorname{tr}\left(\mathbf{p} \mathbf{p}^{\mathrm{H}}\right)\right\}=\operatorname{tr}\left(\mathbf{W}^{2}\left(\sigma_{\mathrm{x}}^{2} \mathbf{H} \mathbf{H}^{\mathrm{H}}+\sigma_{n_{\mathrm{R}}}^{2} \mathbf{I}_{K}\right)\right) \leq P_{\mathrm{R}} .
$$

In the paper, the perfect synchronization is assumed to be achieved at all channel links in the MIMO-AF multiple-relay system. Thus, the received signal vector $\mathbf{r}$ at the destination node is given by

$$
\begin{aligned}
\mathbf{r} & =\mathbf{G} \mathbf{p}+\mathbf{n}_{\mathrm{D}} \\
& =\mathbf{G W H x}+\mathbf{G W} \mathbf{n}_{\mathrm{R}}+\mathbf{n}_{\mathrm{D}},
\end{aligned}
$$

where $\mathbf{G}=\left[\mathbf{g}_{(R(1), f(1))}, \mathbf{g}_{(R(2), f(2))}, \ldots, \mathbf{g}_{(R(K), f(K))}\right] \in C^{N_{\mathrm{D}} \times K}$ in (8) denotes the compound forward channel matrix which is generated by the selected $K$ antennas at the secondtime slot. Note that the selection of a set of $K$ antennas is performed on a total of $K N_{\mathrm{R}}$ possible antenna choices which is composed by $L_{\mathrm{R}}$ candidate relays with a total of the $K$-activated antennas in time-slot I each candidate relay containing $N_{\mathrm{R}}$ corresponding antenna selection choices. The subscript of the pair $(R(k), f(k)), k=1,2, \ldots, K$, represents the selected antenna index $f(k)$ and the corresponding relay index $R(k)$ in time-slot II. $\mathbf{n}_{\mathrm{D}} \in C^{N_{\mathrm{D}} \times 1}$ denotes the ZMCSCG noise vector with the covariance matrix $\mathbf{R}_{\mathbf{n}_{\mathrm{D}}}=E\left\{\mathbf{n}_{\mathrm{D}} \mathbf{n}_{\mathrm{D}}^{\mathrm{H}}\right\}=$ $\sigma_{n_{\mathrm{D}}}^{2} \mathbf{I}_{N_{\mathrm{D}}}$. Remarkably, with the aid of the proposed two-layer antenna selection scheme, an exhaustive antenna-pair search from a pool of $N N_{\mathrm{R}}^{2}$ can be obviated successfully. Finally, (9) can be reformulated as

$$
\mathbf{z}=\mathbf{H}_{\mathrm{eq}} \mathbf{x}+\mathbf{n}_{\mathrm{eq}},
$$

where $\mathbf{H}_{\mathrm{eq}}=\mathbf{G W H}$ and $\mathbf{n}_{\mathrm{eq}}=\mathbf{G W} \mathbf{n}_{\mathrm{R}}+\mathbf{n}_{\mathrm{D}}$ are, respectively, the equivalent channel matrix and the compound noise vector. The capacity of the equivalent channel matrix $\mathbf{H}_{\mathrm{eq}}$ from the source to the destination is represented as

$$
\mathrm{C}_{\mathrm{SD}}\left(\mathbf{H}_{\mathrm{eq}}\right)=\frac{1}{2} \log _{2}\left[\operatorname{det}\left(\mathbf{I}_{N_{\mathrm{D}}}+\frac{P_{\mathrm{s}}}{N_{\mathrm{s}}} \mathbf{H}_{\mathrm{eq}} \mathbf{H}_{\mathrm{eq}}^{\mathrm{H}} \mathbf{Y}^{-1}\right)\right],
$$

where $\boldsymbol{Y}=E\left\{\mathbf{n}_{\mathrm{eq}} \mathbf{n}_{\mathrm{eq}}^{\mathrm{H}}\right\}=\sigma_{n_{\mathrm{D}}}^{2} \mathbf{I}_{N_{\mathrm{D}}}+\sigma_{n_{\mathrm{R}}}^{2} \mathbf{G W}(\mathbf{G W})^{\mathrm{H}}$ denotes the covariance matrix of the compound noise vector $\mathbf{n}_{\mathrm{eq}}$ and the factor $1 / 2$ is multiplied in (11) due to the use of a two-hop relaying transmission.

\section{Modified Greedy Capacity Maximization Algorithm}

An efficient two-layered greedy capacity maximization (GCM) antenna selection technique is proposed for a dualhop non-regenerative MIMO-AF multiple-relay system with multiple antennas equipped at each node in correlated fading channels. This modified GCM (MGCM) antenna selection strategy is employed to select a subset of antenna pairs from multiple relays based on the channel capacity maximization criterion subject to an optimal power allocation across the activated antenna pairs. The use of the proposed twostage MGCM antenna selection is capable of trading in a relatively small fraction of loss in system performance, as compared with existing antenna selection schemes for halfduplex AF-MIMO relay networks, for a considerable reduction in system hardware and signal processing complexity. Let $\mathbf{H}_{i}=\left[\mathbf{h}_{(R(1), b(1))}^{\top}, \mathbf{h}_{(R(2), b(2))}^{\top}, \ldots, \mathbf{h}_{(R(i), b(i))}^{\top}\right]^{\top}$ denote the selected partial backward channel matrix including the $i$ selected antennas. At the $(i+1)$ th step, the capacity of the channel matrix $\mathbf{H}_{i+1}$ with the inclusion of the channel vector $\mathbf{h}_{\left(R_{k}, m\right)}$ from the source node to the $m$ th candidate antenna of the $R_{k}$ th relay can be reexpressed as

$$
\begin{aligned}
& C_{\mathrm{SR}}\left(\mathbf{H}_{i+1}\right) \\
& =\log _{2}\left[\operatorname{det}\left(\mathbf{I}_{i}+\frac{P_{\mathrm{s}}}{N_{\mathrm{s}}} \mathbf{H}_{i} \mathbf{H}_{i}^{\mathrm{H}}\right)\right] \\
& \quad+\log _{2}\left[\operatorname { d e t } \left(1+\frac{P_{\mathrm{s}}}{N_{\mathrm{s}}}\left\|\mathbf{h}_{\left(R_{k}, m\right)}\right\|-\frac{P_{\mathrm{s}}}{N_{\mathrm{s}}} \mathbf{h}_{\left(R_{k}, m\right)}\right.\right. \\
& \left.\left.\quad \times \mathbf{H}_{i}^{\mathrm{H}}\left(\mathbf{I}_{i}+\frac{P_{\mathrm{s}}}{N_{\mathrm{s}}} \mathbf{H}_{i} \mathbf{H}_{i}^{\mathrm{H}}\right)^{-1} \mathbf{H}_{i} \mathbf{h}_{\left(R_{k}, m\right)}^{\mathrm{H}}\right)\right] .
\end{aligned}
$$

The $(i+1)$ th antenna is selected based on the maximization of (13) as follows:

$$
\begin{aligned}
&(R(i+1), b(i+1)) \\
&=\arg \max _{\left(R_{k}, m\right)} \times\left[1+\frac{P_{\mathrm{s}}}{N_{\mathrm{s}}}\left\|\mathbf{h}_{\left(R_{k}, m\right)}\right\|-\frac{P_{\mathrm{s}}}{N_{\mathrm{s}}} \mathbf{h}_{\left(R_{k}, m\right)}\right. \\
&\left.\times \mathbf{H}_{i}^{\mathrm{H}}\left(\mathbf{I}_{i}+\frac{P_{\mathrm{s}}}{N_{\mathrm{s}}} \mathbf{H}_{i} \mathbf{H}_{i}^{\mathrm{H}}\right)^{-1} \mathbf{H}_{i} \mathbf{h}_{\left(R_{k}, m\right)}^{\mathrm{H}}\right] .
\end{aligned}
$$

This selection procedure is repeated until a total of $K$ backward antennas are determined. A total of $N N_{R}$ possible antenna choices need to be examined at the first stage. At the $(i+1)$ th step, the equivalent channel capacity resulted from the antenna pair $\left(R_{k}, m, n\right)$ can be expressed as

$$
\begin{aligned}
\mathrm{C}_{\mathrm{SD}}\left(\mathbf{H}_{i+1}\right) & \\
= & \frac{1}{2} \log _{2}\left[\operatorname{det}\left(\mathbf{\Upsilon}_{i}+w_{\left(R_{k}, m, n\right)}^{2} \mathbf{g}_{\left(R_{k}, n\right)} \mathbf{g}_{\left(R_{k}, n\right)}^{\mathrm{H}}+\frac{P_{\mathrm{S}}}{N_{\mathrm{S}}} \mathbf{Q}_{i} \mathbf{Q}_{i}^{\mathrm{H}}\right)\right] \\
& -\frac{1}{2} \log _{2}\left[\operatorname{det}\left(\mathbf{\Upsilon}_{i}+w_{\left(R_{k}, m, n\right)}^{2} \mathbf{g}_{\left(R_{k}, n\right)} \mathbf{g}_{\left(R_{k}, n\right)}^{\mathrm{H}}\right)\right],
\end{aligned}
$$

where $\mathbf{Q}_{i}=\mathbf{G}_{i} \mathbf{W}_{i} \mathbf{H}_{i}+w_{\left(R_{k}, m, n\right)}^{2} \mathbf{g}_{\left(R_{k}, n\right)} \mathbf{h}_{\left(R_{k}, m\right)}$. According to (15), the antenna pair that is capable of maximizing the 
capacity $\mathrm{C}_{\mathrm{SD}}$ is selected. For simpler selection, we rewrite the selection criterion and select the antenna pair which satisfies

$$
\begin{aligned}
(R(i+1), b(i+1), f(i+1)) & \\
= & \arg \max _{\left(R_{k}, m, n\right)} \operatorname{det} \times\left(\mathbf{A}_{i}+\sum_{a=1}^{4} s_{a} \mathbf{q}_{a} \mathbf{q}_{a}^{\mathrm{H}}\right)-\operatorname{det}\left(\mathbf{\Upsilon}_{i}\right) \\
& -\left(1+w_{\left(R_{k}, m, n\right)}^{2} \mathbf{g}_{\left(R_{k}, n\right)}^{\mathrm{H}} \mathbf{Y}_{i}^{-1} \mathbf{g}_{\left(R_{k}, n\right)}\right),
\end{aligned}
$$

where $\mathbf{q}_{1}=\mathbf{u}_{\left(i, R_{k}, m, n\right)}+\mathbf{g}_{\left(R_{k}, n\right)}, \mathbf{q}_{2}=\mathbf{g}_{\left(R_{k}, n\right)}, \mathbf{q}_{3}=\mathbf{u}_{\left(i, R_{k}, m, n\right)}$, $\mathbf{q}_{4}=w_{\left(R_{k}, m, n\right)}^{2}\left(\left(P_{\mathrm{s}} / N_{\mathrm{s}}\right)\left\|\mathbf{h}_{\left(R_{k}, m\right)}\right\|^{2}+1\right) \mathbf{g}_{\left(R_{k}, n\right)},\left[s_{1}, s_{2}, s_{3}, s_{4}\right]=$ $[1,-1,-1,1], \mathbf{u}_{\left(i, R_{k}, m, n\right)}=\left(P_{\mathrm{s}} / N_{\mathrm{s}}\right) \mathbf{G}_{i} \mathbf{W}_{i} \mathbf{H}_{i} w_{\left(R_{k}, m, n\right)}^{2} \mathbf{h}_{\left(R_{k}, m\right)}^{\mathrm{H}}$, and $\mathbf{A}_{i}=\mathbf{\Upsilon}_{i}+\left(P_{\mathrm{s}} / N_{\mathrm{s}}\right) \mathbf{G}_{i} \mathbf{W}_{i} \mathbf{H}_{i}^{2} \mathbf{W}_{i}^{\mathrm{H}} \mathbf{G}_{i}^{\mathrm{H}}$. Note that only $K N_{\mathrm{R}}$ antenna combinations have to be evaluated at the second stage. It is readily known that the GCM technique proposed in [17] is able to produce an optimal set of active antennas by performing an exhaustive search from a pool of $N N_{\mathrm{R}}^{2}$ antenna combinations to maximize (11). However, the computational cost incurred by such a brute-force search is very expensive and thus leads to a limited usage in practice. This computational requirement is, in general, prohibitive for low-power and cost-effective MIMO-relaying networks. Fortunately, the MGCM approach obviates the necessity of an exhaustive relay and antenna joint search and thus a substantial saving in complexity can be achieved. The fact that in contrast to the GCM approach, the antenna combinations to be treated in the MGCM algorithm are reduced greatly from $N N_{\mathrm{R}}^{2}$ to $(N+K) N_{\mathrm{R}}$ should be pointed out.

\section{Optimal Power Allocation}

In this section, both the optimal and equal-power allocation constraints across the activated $K$ antenna pairs in the halfduplex AF-MIMO multirelay system are considered in what follows. To obtain the optimal-power allocation (OPA) of the relay-weighting matrix $\mathbf{W}$, the singular value decomposition (SVD) technique is employed to perform factorizations of both the compound backward and forward channel matrices, that is,

$$
\begin{aligned}
& \mathbf{H}=\mathbf{U}_{\mathrm{H}} \boldsymbol{\Lambda}_{\mathrm{H}} \mathbf{V}_{\mathrm{H}}^{\mathrm{H}}, \\
& \mathbf{G}=\mathbf{U}_{\mathrm{G}} \boldsymbol{\Lambda}_{\mathrm{G}} \mathbf{V}_{\mathrm{G}}^{\mathrm{H}},
\end{aligned}
$$

where $\mathbf{U}_{\mathrm{H}} \in C^{K \times K}$ and $\mathbf{U}_{\mathrm{G}} \in C^{N_{\mathrm{D}} \times N_{\mathrm{D}}}$ denote the left singular matrices of compound matrices $\mathbf{H}$ and $\mathbf{G}$, respectively. $\mathbf{V}_{\mathbf{H}} \in$ $C^{N_{\mathrm{S}} \times N_{\mathrm{S}}}$ and $\mathbf{V}_{\mathrm{G}} \in C^{K \times K}$ indicate the right singular matrices of compound matrices $\mathbf{H}$ and $\mathbf{G}$, respectively. $\boldsymbol{\Lambda}_{\mathrm{H}}$ and $\boldsymbol{\Lambda}_{\mathrm{G}}$ are diagonal singular-value matrices of compound matrices $\mathbf{H}$ and $\mathbf{G}$, respectively. $\sigma_{\mathrm{H}, i}, i=1,2, \ldots, K$, and $\sigma_{\mathrm{G}, i}, i=$ $1,2, \ldots, K$, represent, respectively, the $i$ th diagonal elements of $\boldsymbol{\Lambda}_{\mathrm{H}}$ and $\boldsymbol{\Lambda}_{\mathrm{G}}$, which are arranged in a decreasing order. Note that the condition of $K \leq \min \left\{N_{\mathrm{S}}, N_{\mathrm{D}}\right\}$ is preserved in what follows. According to [25], the SNR at the destination node is readily shown as

$$
\mathrm{SNR}=\frac{\operatorname{tr}\left(\mathbf{G W H} \sigma_{\mathbf{x}}^{2} \mathbf{H}^{\mathrm{H}} \mathbf{W}^{\mathrm{H}} \mathbf{G}^{\mathrm{H}}\right)}{\operatorname{tr}\left(\mathbf{G W} \sigma_{n_{\mathrm{R}}}^{2} \mathbf{W}^{\mathrm{H}} \mathbf{G}^{\mathrm{H}}\right)+\sigma_{n_{\mathrm{D}}}^{2}} .
$$

The SNR optimization problem under the total transmit power constraint on $K$-activated antenna pairs can be equivalently formulated as

$$
\begin{aligned}
& \min _{w_{i, i}^{2}}-\sum_{i=1}^{K} \frac{\sigma_{\mathrm{G}, i}^{2} \sigma_{\mathrm{H}, i}^{2} \sigma_{\mathrm{x}}^{2} w_{i, i}^{2}}{\sigma_{\mathrm{G}, i}^{2} \sigma_{n_{\mathrm{R}}}^{2} w_{i, i}^{2}+\sigma_{n_{\mathrm{D}}}^{2}} \\
& \text { s.t. } \sum_{i=1}^{K} w_{i, i}^{2}\left(\sigma_{\mathrm{x}}^{2} \sigma_{\mathrm{H}, i}^{2}+\sigma_{n_{\mathrm{R}}}^{2}\right) \leq P_{\mathrm{R}}
\end{aligned}
$$

The optimum values of $w_{i, i}^{2}, i=1,2, \ldots, K$, can be attained based on the Karush-Kuhn-Tucker (KKT) optimality conditions [26], which is given by

$$
\begin{array}{r}
w_{i, i}^{2}=\left(\frac{\left(\sigma_{\mathrm{G}, i} \sigma_{\mathrm{H}, i} \sigma_{\mathrm{x}} \sigma_{n_{\mathrm{R}}} / \lambda^{1 / 2} \sqrt{\sigma_{\mathrm{x}}^{2} \sigma_{\mathrm{H}, i}^{2}+\sigma_{n_{\mathrm{R}}}^{2}}\right)-\sigma_{n_{\mathrm{D}}}^{2}}{\sigma_{\mathrm{G}, i}^{2} \sigma_{n_{\mathrm{R}}}^{2}}\right)_{+}, \\
i=1,2, \ldots, K,
\end{array}
$$

where the Lagrangian multiplier $\lambda$ is chosen to meet the total transmit-power constraint over $K$ antenna pairs in (18), which is determined by means of an iterative water-filling technique as follows:

$$
\lambda=\left(\sum_{i=1}^{K} \frac{\sigma_{\mathrm{G}, i} \sigma_{\mathrm{H}, i} \sigma_{\mathrm{x}} \sigma_{n_{\mathrm{D}}}\left(\sigma_{\mathrm{H}, i}^{2} \sigma_{\mathrm{x}}^{2}-\sigma_{n_{\mathrm{D}}}^{2}\right)}{P_{\mathrm{R}} \sigma_{\mathrm{G}, i}^{2} \sigma_{n_{\mathrm{R}}}^{2} \sqrt{\sigma_{\mathrm{x}}^{2} \sigma_{\mathrm{H}, i}^{2}+\sigma_{n_{\mathrm{R}}}^{2}}}\right)^{2} .
$$

When the equal-power allocation (EPA) constraints across the activated $K$ antenna pairs is considered, the values of $w_{i, i}^{2}, i=1,2, \ldots, K$, can be attained as

$$
w_{i, i}^{2}=\frac{P_{\mathrm{R}}}{K\left(\sigma_{\mathrm{x}}^{2}\left\|\mathbf{h}_{(R(i), b(i))}\right\|_{\mathrm{F}}^{2}+\sigma_{n_{\mathrm{R}}}^{2}\right)}, \quad i=1,2, \ldots, K .
$$

\section{Destination Antenna Subset Selection Criteria}

To alleviate hardware cost and implementation complexity of a dual-hop AF-wireless MIMO multiple-relay system, antenna selection schemes are also performed at the destination node. Through the use of (11), the capacity of the equivalent channel matrix $\mathbf{H}_{\mathrm{eq}}$ from the source to the destination can be reformulated as

$$
\mathrm{C}_{\mathrm{SD}}\left(\mathbf{H}_{\mathrm{eq}}\right)=\frac{-1}{2} \log _{2}\left[\operatorname{det}\left(\mathbf{I}_{N_{\mathrm{D}}}+\frac{P_{\mathrm{s}}}{N_{\mathrm{s}}} \mathbf{H}_{\mathrm{eq}} \mathbf{H}_{\mathrm{eq}}^{\mathrm{H}} \mathbf{\Upsilon}^{-1}\right)^{-1}\right] \text {. }
$$

The MSE for the $j$ th detection stage of the dual-hop AFMIMO multiple-relay system can be expressed as [27]

$$
\mathrm{MSE}_{k_{j}}=\left[\frac{P_{\mathrm{s}}}{N_{\mathrm{s}}}\left(\mathbf{I}_{N_{\mathrm{D}}}+\overline{\mathbf{H}}_{\mathrm{eq}_{j}} \overline{\mathbf{H}}_{\mathrm{eq}_{j}}^{\mathrm{H}} \mathbf{Y}_{\mathrm{eq}_{j}}^{-1}\right)^{-1}\right]_{k_{j}, k_{j}},
$$

where the matrix $\overline{\mathbf{H}}_{\mathrm{eq}_{j}}$ in (23) is obtained by setting all the $k_{1}$ th, $k_{2}$ th, ..., $k_{(j-1)}$ th columns of $\mathbf{H}_{\mathrm{eq}}$ to zeros. By recursively 
substituting the $\mathrm{MSE}_{k_{j}}$ of (23) at stage $k_{j}, k_{j}=1,2, \ldots, N_{\mathrm{D}}$, into (22), the capacity of the dual-hop AF-MIMO multiplerelay system at the final detection stage $N_{D}$ can be reexpressed as [27]

$$
\begin{aligned}
& \mathrm{C}_{\mathrm{SD}}\left(\mathbf{H}_{\mathrm{eq}}\right) \\
& =-\frac{P_{\mathrm{s}}}{2 N_{\mathrm{s}}} \log _{2}\left(\prod_{k_{j}=1}^{i} \mathrm{MSE}_{k_{j}} \times \operatorname{det}\left(\mathbf{I}_{N_{\mathrm{D}}-i}+\overline{\mathbf{H}}_{\mathrm{eq}_{i}} \overline{\mathbf{H}}_{\mathrm{eq}_{i}}^{\mathrm{H}} \mathbf{Y}_{\mathrm{eq}_{i}}^{-1}\right)^{-1}\right) \\
& =-\frac{P_{\mathrm{s}}}{2 N_{\mathrm{s}}} \log _{2}\left(\prod_{k_{j}=1}^{N_{\mathrm{D}}} \mathrm{MSE}_{k_{j}}\right) .
\end{aligned}
$$

Thus, according to (24), to maximize the capacity of the selected antenna subset $c_{l}$ for the dual-hop AF-MIMO multiple-relay system is equivalent to minimize the products of MSEs at all stages (i.e., $\mathrm{MSE}_{k_{j}}, k_{j}=1,2, \ldots, N_{\mathrm{D}}$ ), for $c_{l}$. In what follows, let $c_{l}$, for $l=1,2, \ldots,\left(\begin{array}{c}N_{\mathrm{D}} \\ L\end{array}\right)$, denote all possible combinations of $L$ antennas out of the complete set of $N_{\mathrm{D}}$ receive antennas. In the sequel, the symbol detection is performed by the V-BLAST MMSE detection scheme.

\section{Existing Antenna Subset Selection Criteria}

In this section, two categories of joint relay-and-antenna selection algorithms, namely, distributed orthogonal relay selection (DORS) [11] and fast antenna subset selection algorithm (FASSA) [13], are briefly reviewed.

6.1. Distributed Orthogonal Relay Selection. The first relaybased antenna selection algorithm is called "distributed orthogonal relay selection" (DORS) [11]. The authors in [11] propose a selection process that combines projection and harmonic mean to select antenna pairs from multiplerelay nodes. In order to maximize the channel capacity, two selection criteria play significant roles. At first, the equivalent channel matrix has to be made as diagonal as possible to minimize the interferences among the parallel data streams. Secondly, the equivalent channel gains need to be as large as possible to maximize the SNR per data stream. Let $\mathbf{H}_{i}=\left[\mathbf{h}_{(R(1), b(1))}, \mathbf{h}_{(R(2), b(2))}, \ldots, \mathbf{h}_{(R(i), b(i))}\right]^{\top}$ and $\mathbf{G}_{i}=\left[\mathbf{g}_{(R(1), f(1))}, \mathbf{g}_{(R(2), f(2))}, \ldots, \mathbf{g}_{(R(i), f(i))}\right]$ denote the backward and forward channel matrices including the $i$-selected antennas-respectively, and the index $\left(R_{k}, m, n\right)$ represents the $m$ th backward candidate antenna and the $n$th forward candidate antenna at the $R_{k}$ th relay. The $(i+1)$ th antenna pair is determined by

$$
\begin{aligned}
& (R(i+1), b(i+1), f(i+1)) \\
& \quad=\arg \max _{\left(R_{k}, m, n\right)} f\left(\left\|\mathbf{h}_{\left(R_{k}, m\right)}^{\perp}\right\|_{\mathrm{F}},\left\|\mathbf{g}_{\left(R_{k}, n\right)}^{\perp}\right\|_{\mathrm{F}}\right),
\end{aligned}
$$

where $f\left(\|\mathbf{a}\|_{\mathrm{F}},\|\mathbf{b}\|_{\mathrm{F}}\right)$ denotes a harmonic mean of vectors a and $\mathbf{b}$ given by $f\left(\|\mathbf{a}\|_{\mathrm{F}},\|\mathbf{b}\|_{\mathrm{F}}\right)=\left(2\|\mathbf{a}\|_{\mathrm{F}} \cdot\|\mathbf{b}\|_{\mathrm{F}}\right) /\left(\|\mathbf{a}\|_{\mathrm{F}}+\|\mathbf{b}\|_{\mathrm{F}}\right)$. Additionally, the projection vectors $\mathbf{h}_{\left(R_{k}, m\right)}^{\perp}$ and $\mathbf{g}_{\left(R_{k}, n\right)}^{\perp}$ are derived by means of Gram-Schmidt (GS) orthogonalization. The backward and forward candidate antenna vectors are projected into the spaces spanned by $\mathbf{H}_{i}$ and $\mathbf{G}_{i}$, respectively, which are given by

$$
\begin{aligned}
\mathbf{h}_{\left(R_{k}, m\right)}^{\perp} & =\mathbf{h}_{\left(R_{k}, m\right)}-\sum_{j=1}^{i} \frac{\mathbf{h}_{\left(R_{k}, m\right)} \mathbf{h}_{(R(j), b(j))}^{\mathrm{H}}}{\left\|\mathbf{h}_{(R(j), b(j))}\right\|_{\mathrm{F}}^{2}} \mathbf{h}_{(R(j), b(j))} \\
\mathbf{g}_{\left(R_{k}, n\right)}^{\perp} & =\mathbf{g}_{\left(R_{k}, n\right)}-\sum_{j=1}^{i} \frac{\mathbf{g}_{\left(R_{k}, n\right)} \mathbf{g}_{(R(j), f(j))}^{\mathrm{H}}}{\left\|\mathbf{g}_{(R(j), f(j))}\right\|_{\mathrm{F}}^{2}} \mathbf{g}_{(R(j), f(j)) .}
\end{aligned}
$$

The DORS antenna selection algorithm runs until a subset of $K$ antennas are selected.

6.2. Modified Fast Antenna Subset Selection Algorithm. In [13], a fast antenna subset selection algorithm (FASSA) is introduced for a two-hop MIMO-AF relay system to avoid the intensive complexity of an exhaustive search algorithm and make antenna subset selection practical for applications. The FASSA algorithm for MIMO-AF relay attempts to maximize a lower bound of the capacity instead of the exact capacity. According to [13], the capacity $\mathrm{C}_{S \mathrm{D}}$ in (11) can be further simplified and lower bounded by

$$
\mathrm{C}_{\mathrm{SD}}=\frac{1}{2} \log _{2}\left[\operatorname{det}\left(\mathbf{I}_{\mathrm{K}}+\frac{P_{\mathrm{s}}}{N_{\mathrm{s}}} \mathbf{H} \mathbf{H}^{\mathrm{H}} \mathbf{G}^{\mathrm{H}} \mathbf{G}\left(\mathbf{I}_{\mathrm{K}}+\rho \mathbf{G}^{\mathrm{H}} \mathbf{G}\right)^{-1}\right)\right]
$$

$$
\begin{aligned}
& =\frac{1}{2} \log _{2}\left[\operatorname { d e t } \left(\mathbf{I}_{K}+\frac{P_{\mathrm{s}}}{N_{\mathrm{s}}} \mathbf{H H}^{\mathrm{H}} \mathbf{G}^{\mathrm{H}} \mathbf{G}\right.\right. \\
& \left.\left.\quad \times\left(\mathbf{I}_{\mathrm{K}}-\left(\mathbf{I}_{\mathrm{K}}+\rho \mathbf{G}^{\mathrm{H}} \mathbf{G}\right)^{-1}\right)\right)\right] \\
& \geq \frac{1}{2} \log _{2} \frac{\left(P_{\mathrm{s}} / N_{\mathrm{s}}\right)^{K} \operatorname{det}\left(\mathbf{H} \mathbf{H}^{\mathrm{H}}\right) \operatorname{det}\left(\mathbf{G}^{\mathrm{H}} \mathbf{G}\right)}{\prod_{a=1}^{\mathrm{K}}\left((1 / \rho)+\left\|\mathbf{g}_{(R(a), f(a))}\right\|^{2}\right)}
\end{aligned}
$$

where the coefficient $\rho$ keeps the total transmit power of the $L_{\mathrm{R}}$-selected relays equal to $P_{\mathrm{R}}$. It should be noticed that the lower bound of the capacity $\mathrm{C}_{\mathrm{SD}}$ is derived based on the Hadamard inequality [28]. In the $(n+1)$ th step of the FASSA algorithm, the index set of $(R(i+1), b(i+1), f(i+1))$ is determined in order to maximize the lower bound given in (29), which satisfies

$$
\begin{aligned}
(R(i+1), & b(i+1), f(i+1)) \\
= & \arg \max _{\left(R_{k}, m, n\right)} \operatorname{det}\left(\left[\mathbf{H} \cup \mathbf{h}_{\left(R_{k}, m\right)}\right]\left[\mathbf{H} \cup \mathbf{h}_{\left(R_{k}, m\right)}\right]^{\mathrm{H}}\right) \\
& \frac{\operatorname{det}\left(\left[\mathbf{G} \cup \mathbf{g}_{\left(R_{k}, n\right)}\right]^{\mathrm{H}}\left[\mathbf{G} \cup \mathbf{g}_{\left(R_{k}, n\right)}\right]\right)}{\prod_{a=1}^{i+1}\left((1 / \rho)+\left\|\mathbf{g}_{(R(a), f(a))}\right\|^{2}\right)} .
\end{aligned}
$$




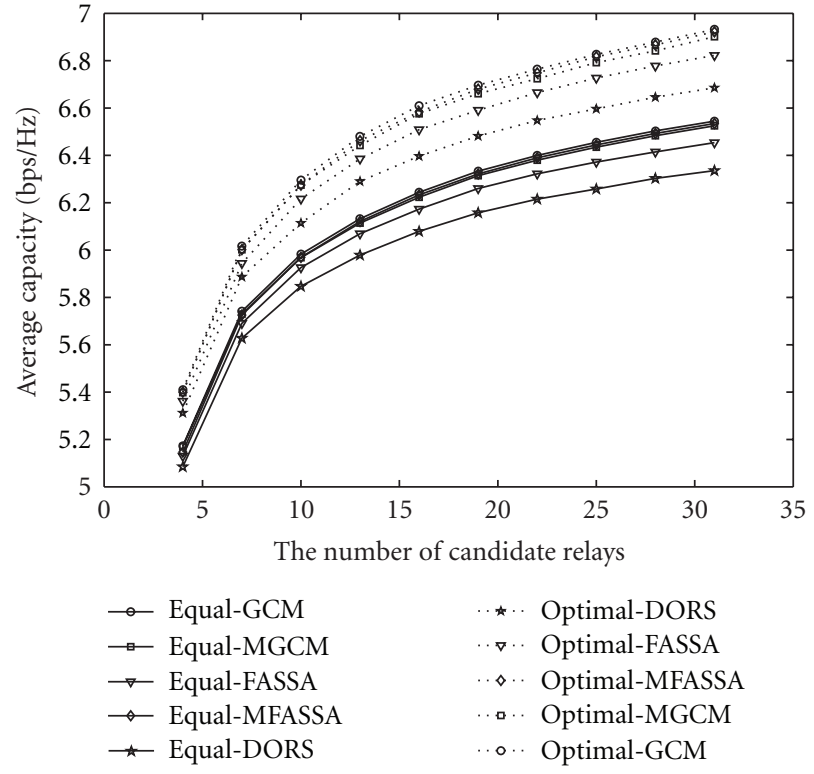

(a)

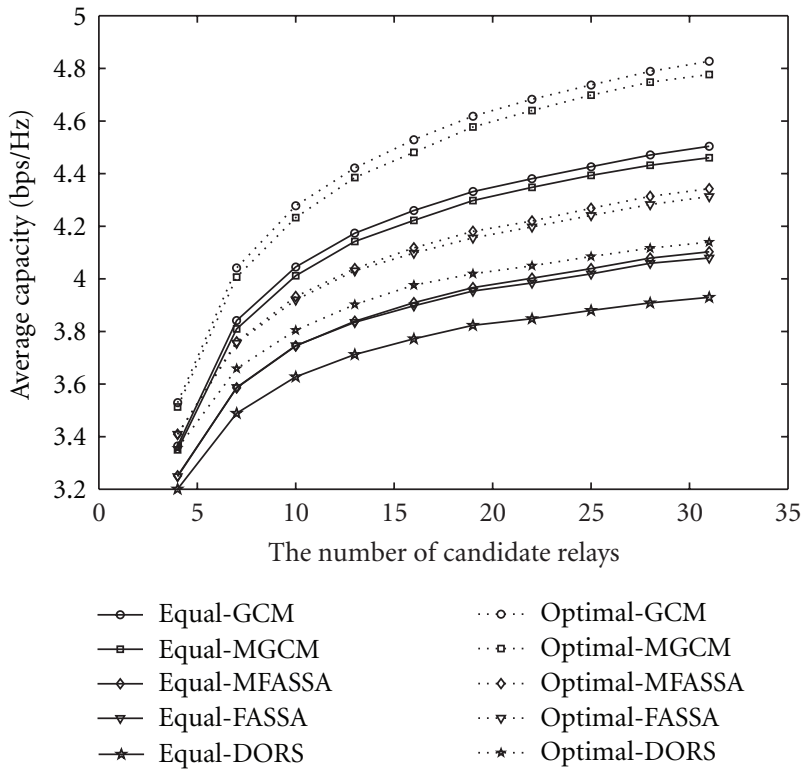

(b)

FIGURE 2: System-capacity performance comparisons of various relay antenna selection algorithms in terms of the number of candidate relays $N$ for an AF-MIMO multiple-relay system with the use of $\left[N_{S}, N_{\mathrm{R}}, N_{\mathrm{D}}, K\right]=[10,2,10,4]$ and the (a) low and (b) high spatial-correlation scenarios under either an optimal or equal-power allocation.

However, the FASSA scheme in [13] ignores the effects of relay weighting matrix when executing antenna selection so that the accuracy of channel capacity is affected seriously. Thus, by taking the relay weighting matrix into account, the modified FASSA (MFASSA) algorithm can be operated in more comprehensive and accurate way. Let $\mathbf{W}_{i}$ denote the compound weighting matrix at the ith step and then be substituted into (29). After the completeness of the $i$ th antenna selection, the lower bound for $\mathrm{C}_{\mathrm{SD}}$ shown in (29) can be reformulated as

$$
\mathrm{C}_{\mathrm{SD}} \geq \frac{1}{2} \log _{2} \frac{\left(P_{\mathrm{s}} / N_{\mathrm{s}}\right)^{i} \operatorname{det}\left(\mathbf{H}_{i} \mathbf{H}_{i}^{\mathrm{H}}\right) \operatorname{det}\left(\left(\mathbf{G}_{i} \mathbf{W}_{i}\right)^{\mathrm{H}}\left(\mathbf{G}_{i} \mathbf{W}_{i}\right)\right)}{\prod_{a=1}^{i}\left(\sigma_{n_{\mathrm{D}}}^{2} \mathbf{I}_{i}+\sigma_{n_{\mathrm{R}}}^{2}\left(\mathbf{G}_{i} \mathbf{W}_{i}\right)^{\mathrm{H}}\left(\mathbf{G}_{i} \mathbf{W}_{i}\right)\right)_{a, a}} .
$$

\section{Numerical Results}

In this section, computer simulations are conducted to demonstrate the system-capacity performance of the proposed antenna-pair selection scheme for a dual-hop AFMIMO multiple-relay system in correlated channels. Let the notation of $\left[N_{S}, N_{\mathrm{R}}, N_{\mathrm{D}}, N, K\right]$ denote a subset of $K$ antenna pairs selected from an AF-mode MIMO $N$-relay system equipped with $N_{S}, N_{R}$, and $N_{D}$ antennas, respectively, at the source, each relay, and the destination. A Kronecker correlated channel model [22] with the use of $d_{\mathrm{S}}=d_{\mathrm{R}_{i}}=d_{\mathrm{D}}$ and $\theta_{\mathrm{S}}=\theta_{\mathrm{R}_{i}}=\theta_{\mathrm{D}}$ is considered in the correlated MIMO channel. Here $d$ denotes the antenna spacing and $\theta$ stands for the angular spread. Throughout simulations, both the total transmit power of $K$-activated antenna pairs selected from $L_{\mathrm{R}}$ relays (i.e., $\left.L_{\mathrm{R}} \leq K\right) P_{\mathrm{R}}$ and the power constraint at the source $P_{\mathrm{S}}$ are set to $10 \mathrm{~dB}$. In addition, it is assumed that the full channel state information (CSI) of all channel links is available and the perfect synchronization can be achieved. Here, 10000 Monte Carlo runs are conducted for each relay deployment.

In Figure 2, the system-capacity comparisons of various antenna subset selection schemes are provided for an AF-MIMO multiple-relay system with the use of $\left[N_{\mathrm{S}}, N_{\mathrm{R}}, N_{\mathrm{D}}, K\right]=[10,2,10,4]$ and two different spatialcorrelation scenarios, that is, $d_{\mathrm{S}}=d_{\mathrm{R}_{i}}=d_{\mathrm{D}}=0.8 \lambda$ and $\theta_{\mathrm{S}}=\theta_{\mathrm{R}_{i}}=\theta_{\mathrm{D}}=100^{\circ}$ in (a) and $d_{\mathrm{S}}=d_{\mathrm{R}_{i}}=d_{\mathrm{D}}=0.4 \lambda$ and $\theta_{\mathrm{S}}=\theta_{\mathrm{R}_{i}}=\theta_{\mathrm{D}}=40^{\circ}$ in (b), in terms of the number of candidate relays. It is observed from both figures that the system-capacity performance degrades significantly when all antenna-pair selection schemes operate in a high spatial-correlation channel. Among those five antenna selection algorithms, the GCM method accomplishes the best capacity performance at the expense of the most heavy computational complexity. Remarkably, it is evident that the proposed MGCM antenna selection scheme is capable of achieving similar capacity level to the GCM algorithm at a much lower complexity cost. It is also seen from Figure 2 that the proposed MGCM method produces uniformly better performance than those of the FASSA, the MFASSA, and the DORS techniques. Moreover, the DORS scheme produces a worst capacity performance among those antenna selection schemes due to ignorance of the effects of the matrix $Y$ during the antenna selection process. Furthermore, it should be emphasized that all antenna-pair selection algorithms with the aid of an optimal power allocation outperform significantly the ones with an equal power allocation in system capacity performance no matter under the low or high correlated channel. 


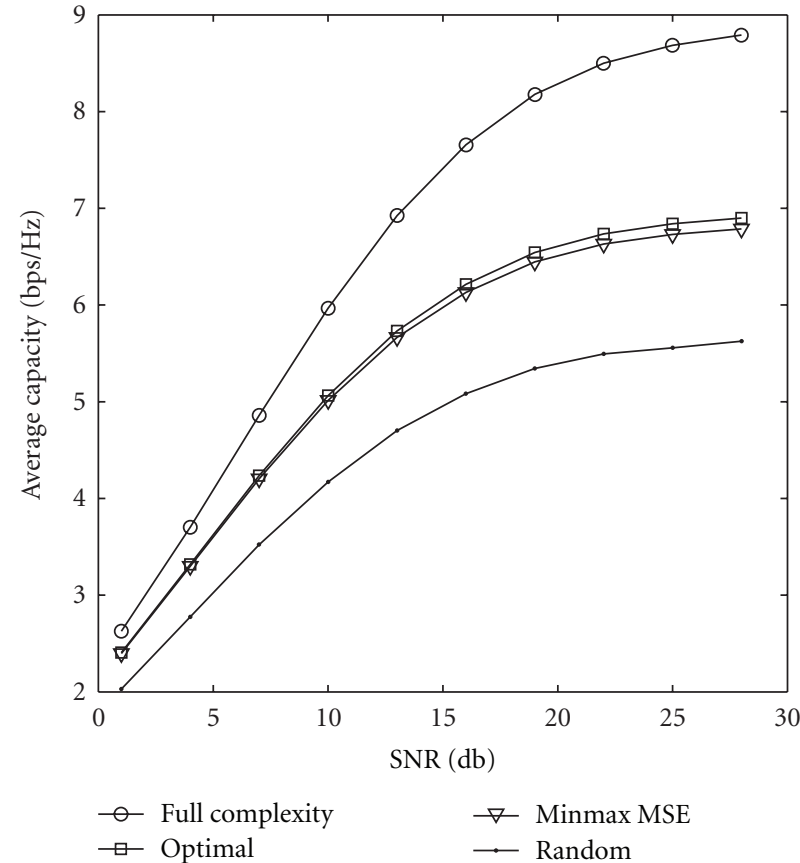

(a)

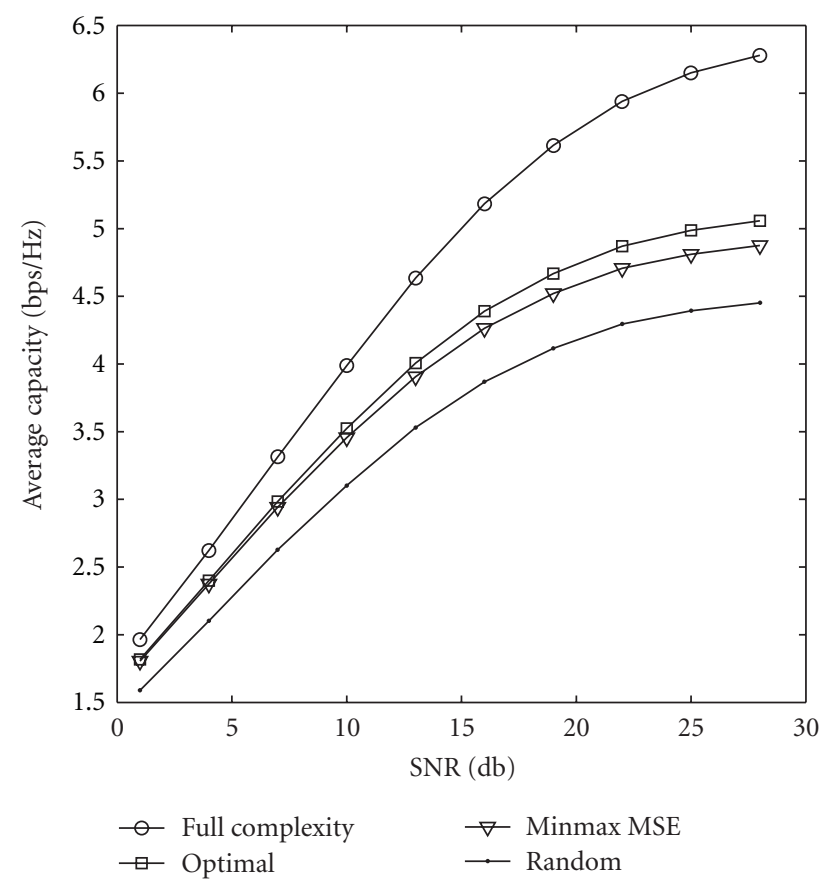

(b)

FIGURE 3: System-capacity performance comparisons of various destination antenna selection algorithms in terms of SNR for an AF-MIMO multiple-relay system with $\left[N_{S}, N_{\mathrm{R}}, N_{\mathrm{D}}, N, K, L\right]=[10,2,10,10,4,4]$ and a fixed relay antenna-pair selection process in the (a) low and (b) high spatial-correlation channels.

Figure 3 evaluates system-capacity comparisons of various destination antenna selection algorithms for an AFMIMO multiple-relay system with $\left[N_{\mathrm{S}}, N_{\mathrm{R}}, N_{\mathrm{D}}, N, K, L\right]=$ $[10,2,10,10,4,4]$ and a fixed relay antenna-pair selection process in the low (a) and high (b) spatial-correlation channels in terms of SNR. In the figure, "full complexity" means that all the available receive antennas at the destination node are employed for demodulation in an AF-MIMO multiplerelay system (i.e., the full set of antennas at the destination node). "Random" denotes the receive antenna subset is chosen randomly from an entire set of receive antennas. "MinmaxMSE" indicates that the desired receive antennas are selected based on the performance-based criterion in Section 5 which is determined by the calculated MSEs in (24). "Optimal" represents that an exhaustive search over all possible receive-antenna combinations is performed based on the capacity-based criterion. As the results in Figure 2, the system-capacity performance degrades significantly when all destination antenna selection schemes operate in a high spatial-correlation channel. In addition, from both figures, the proposed antenna selection algorithm at destination can maintain good performance no matter in high or low correlation channel. The fact that with the use of moderate antenna selection criteria, the selected receive antenna subset is able to produce an improved channel capacity over the random antenna selection should be emphasized.

\section{Conclusions}

In this paper, a reduced-complexity layered MGCM antenna subset selection scheme is proposed for a dual-hop AFMIMO multiple-relay system over correlated fading channels. From simulation results, it is obvious that a considerable reduction in system hardware cost and signal processing complexity is achieved by means of the modified GCM architecture under either optimal or equal-power allocation. In addition, the modified GCM method provides similar capacity performance to the GCM scheme and achieves better capacity performance than those of the FASSA, the MFASSA, and the DORS techniques. As a consequence, this computationally-efficient modified GCM-based antennapair selection strategy is suitable for applications in the nonregenerative AF-MIMO multiple-relay wireless systems.

\section{Acknowledgment}

This work was supported by Taiwan National Science Council under Grant NSC98-2221-E-194-018-MY2.

\section{References}

[1] I. E. Telatar, "Capacity of multi-antenna Gaussian channels," European Transactions on Telecommunications, vol. 10, no. 6, pp. 585-595, 1999. 
[2] A. F. Molisch, M. Z. Win, Y.-S. Choi, and J. H. Winters, "Capacity of MIMO systems with antenna selection," IEEE Transactions on Wireless Communications, vol. 4, no. 4, pp. 1759-1772, 2005.

[3] P. W. Wolniansky, G. J. Foschini, G. D. Golden, and R. A. Valenzuela, "V-BLAST: an architecture for realizing very high data rates over the rich-scattering wireless channel," in Proceedings of the International Symposium on Signals, Systems, and Electronics (ISSSE '98), pp. 295-300, September 1998.

[4] A. Sendonaris, E. Erkip, and B. Aazhang, "User cooperation diversity_part I: system description," IEEE Transactions on Communications, vol. 51, no. 11, pp. 1927-1938, 2003.

[5] J. N. Laneman, D. N. C. Tse, and G. W. Wornell, "Cooperative diversity in wireless networks: efficient protocols and outage behavior," IEEE Transactions on Information Theory, vol. 50, no. 12, pp. 3062-3080, 2004.

[6] O. Muñoz-Medina, J. Vidal, and A. Agustín, "Linear transceiver design in nonregenerative relays with channel state information," IEEE Transactions on Signal Processing, vol. 55, no. 6, pp. 2593-2604, 2007.

[7] J. Luo, R. S. Blum, L. J. Cimini, L. J. Greenstein, and A. M. Haimovich, "Decode-and-forward cooperative diversity with power allocation in wireless networks," IEEE Transactions on Wireless Communications, vol. 6, no. 3, pp. 793-799, 2007.

[8] P. Sudarshan, H. Dai, and B. L. Hughes, "Statistics-based antenna selection for multi-access MIMO systems," in Proceedings of the Asilomar Conference on Signals, Systems and Computers, pp. 903-907, Pacific Grove, Calif, USA, November 2004.

[9] D. S. Michalopoulos, G. K. Karagiannidis, T. A. Tsiftsis, and R. K. Mallik, "Distributed transmit antenna selection (DTAS) under performance or energy consumption constraints," IEEE Transactions on Wireless Communications, vol. 7, no. 4, pp. 1168-1173, 2008.

[10] S. W. Peters and R. W. Heath Jr., "Nonregenerative MIMO relaying with optimal transmit antenna selection," IEEE Signal Processing Letters, vol. 15, pp. 421-424, 2008.

[11] W. Zhang and K. B. Letaief, "Opportunistic relaying for dualhop wireless MIMO channels," in Proceedings of the IEEE Global Telecommunications Conference, pp. 3985-3989, New Orleans, La, USA, November 2008.

[12] J.-C. Chen and C.-K. Wen, "Near-optimal relay subset selection for two-way amplify-and-forward MIMO relaying systems," IEEE Transactions on Wireless Communications, vol. 10, no. 1, pp. 37-42, 2011.

[13] G. Zhang, G. Li, and J. Qin, "Fast antenna subset selection algorithms for multiple-input multiple-output relay systems," IET Communications, vol. 3, no. 11, pp. 1693-1703, 2009.

[14] H. Park and J. Chun, "A two-stage antenna subset selection scheme for amplify-and-forward MIMO relay systems," IEEE Signal Processing Letters, vol. 17, no. 11, pp. 953-956, 2010.

[15] Y. Zhang, G. Zheng, C. Ji, K.-K. Wong, D. J. Edwards, and T. Cui, "Near-optimal joint antenna selection for amplifyand-forward relay networks," IEEE Transactions on Wireless Communications, vol. 9, no. 8, pp. 2401-2407, 2010.

[16] M. Ding, S. Liu, H. Luo, and W. Chen, "MMSE based greedy antenna selection scheme for AF MIMO relay systems," IEEE Signal Processing Letters, vol. 17, no. 5, pp. 433-436, 2010.

[17] M. Ding, S. Liu, H. Luo, and X. Wang, "Antenna selection for AF MIMO relay networks and the capacity scaling tendency," in Proceedings of the International Conference on Communications, pp. 1-5, Cape Town, South Africa, May 2010.
[18] G. Amarasuriya, C. Tellambura, and M. Ardakani, "Joint relay and antenna selection for dual-hop amplify-andforward MIMO relay networks," IEEE Transactions on Wireless Communications, vol. 11, no. 2, pp. 493-499, 2012.

[19] G. Amarasuriya, C. Tellambura, and M. Ardakani, "Performance analysis of hop-by-hop beamforming for dual-hop MIMO AF relay networks," IEEE Transactions on Communications, vol. 60, no. 7, pp. 1823-1837, 2012.

[20] G. Amarasuriya, C. Tellambura, and M. Ardakani, "Performance analysis framework for transmit antenna selection strategies of cooperative MIMO AF relay networks," IEEE Transactions on Vehicular Technology, vol. 60, no. 7, pp. 30303044, 2011.

[21] M. K. Özdemir, E. Arvas, and H. Arslan, "Dynamics of spatial correlation and implications on MIMO systems," IEEE Communications Magazine, vol. 42, no. 6, pp. S14-S19, 2004.

[22] D.-S. Shiu, G. J. Foschini, M. J. Gans, and J. M. Kahn, "Fading correlation and its effect on the capacity of multielement antenna systems," IEEE Transactions on Communications, vol. 48, no. 3, pp. 502-513, 2000.

[23] A. V. Zelst and J. S. Hammerschmidt, "A single coefficient spatial correlation model for MIMO radio channels," in Proceedings of the 27th General Assembly of the International Union of Radio Science (URSI '02), pp. 1-4, Maastricht, The Netherlands, August 2002.

[24] G. D. Durgin and T. S. Rappaport, "Effects of multipath angular spread on the spatial cross-correlation of received voltage envelopes," in Proceedings of the IEEE Vehicular Technology Conference, vol. 2, pp. 996-1000, Houston, Tex, USA, May 1999.

[25] A. S. Behbahani, R. Merched, and A. M. Eltawil, "Optimizations of a MIMO relay network," IEEE Transactions on Signal Processing, vol. 56, no. 10, pp. 5062-5073, 2008.

[26] E. K. P. Chong and S. H. Zak, An Introduction to Optimization, John Wiley \& Sons, New York, NY, USA, 2nd edition, 2001.

[27] D. Lu and D. K. C. So, "Performance based receive antenna selection for V-BLAST systems," IEEE Transactions on Wireless Communications, vol. 8, no. 1, pp. 214-225, 2009.

[28] X. Zhang, Matrix Analysis and Applications, Tsinghua University Press, 2004. 

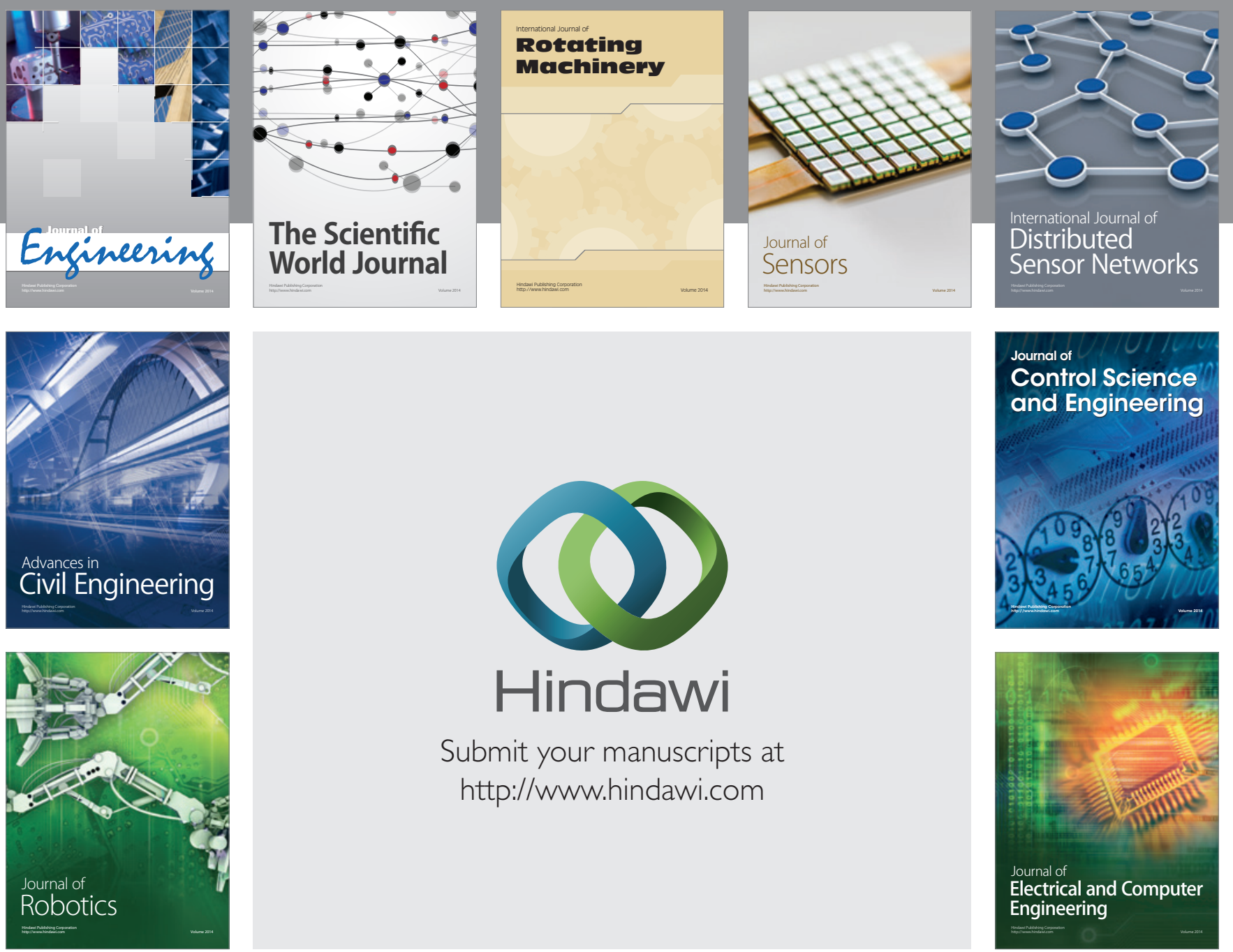

Submit your manuscripts at

http://www.hindawi.com
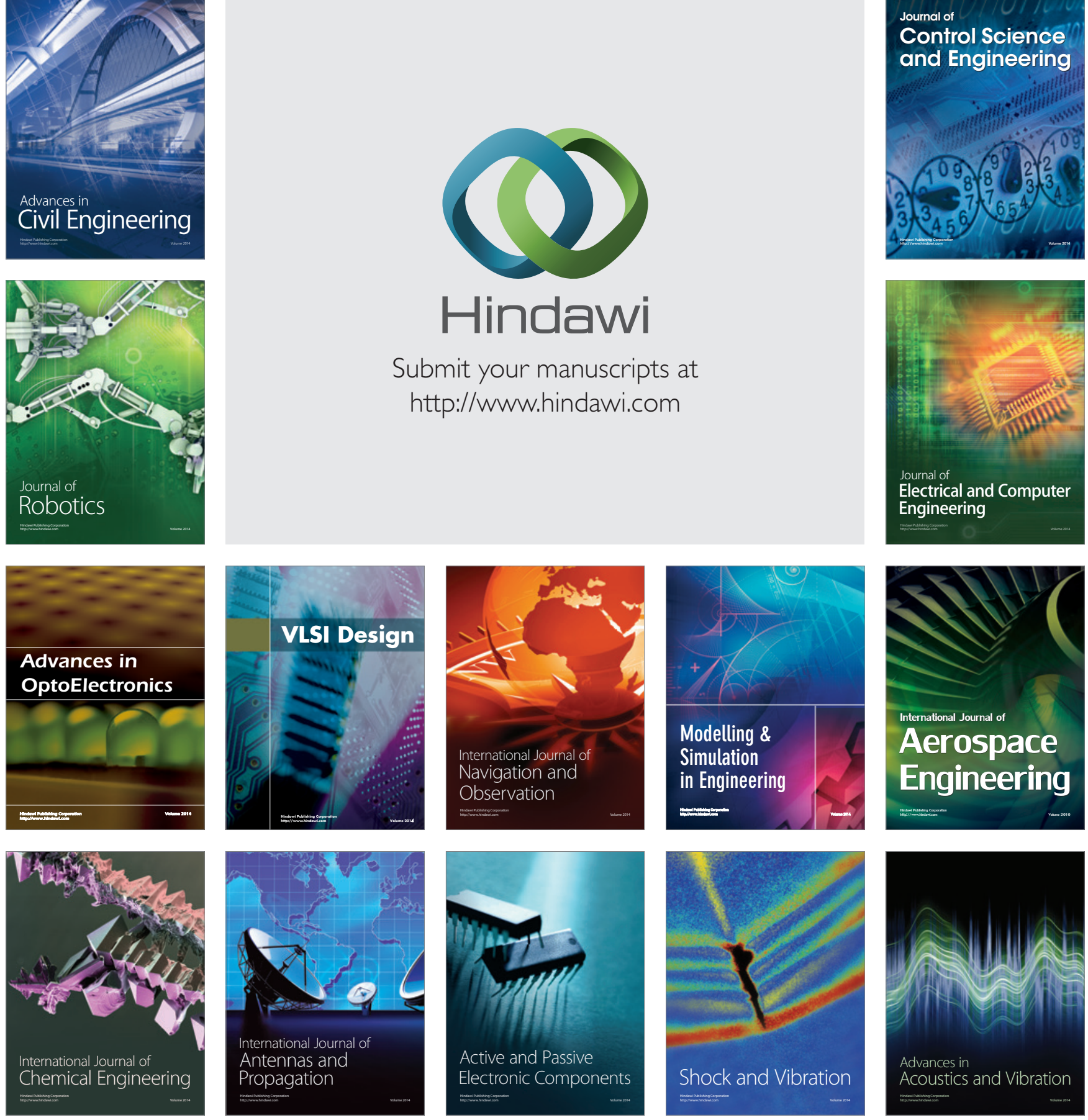